UDC 681.518

DOI: 10.30987/conferencearticle_61c997ee849986.88188810

\title{
GENERAL METHODOLOGY OF EVALUATION AND SELECTION OF COMPONENTS OF AUTOMATED SYSTEMS
}

Okhunov Dilshod Mamatjonovich, candidate of economic sciences

Okhunov Mamatjon Hamidovich, candidate of physical and mathematical sciences

Uzbekistan, Fergana, Ferghana branch of Tashkent University of Information Technologies named after Muhammad al-kHorezmi, mamatdilshod@ rambler.ru

Abstract. The features of modern control systems are: the multifunctional nature of production; the multi-connectivity of processes and tasks of automated systems; the need to ensure interaction with systems of different levels and the same level, etc. The article discusses the methodology of evaluation and selection of components of automated systems.

Keywords: automated system, automated object, economic and mathematical methods and models, information and computing services, expert system, bank of algorithms and procedures, knowledge bank.

In the process of designing automated systems (AS), a wide range of tasks arise for evaluating and selecting AS components: the selection of functions (tasks) to be automated, economic and mathematical methods and models, algorithms, software and hardware that ensure the implementation of these functions; the selection and evaluation of system-wide design solutions (operating systems and programming systems, database management systems, application software packages, etc.); automated workplaces, etc [1].

The purpose of solving the selection tasks is to ensure compliance between the needs of the automated object and the capabilities of the AS created for them. The needs of the object are expressed through the elements of the AS and are as follows:

- compliance with the parameters of the AS characteristic of the automated object;

- compliance of automated functions with the needs of AS users;

- compliance of the used economic and mathematical methods and models and algorithms for solving the problem with automated functions. This correspondence allows the accelerated receipt of the necessary information, saving machine time;

- compliance of the software tools used with the methods and algorithms for solving the problem;

- compliance of the technical means and systems used with the information and software of the AS [2].

The correspondence between the parameters of the AS and the parameters of the designed AS is achieved by an optimal choice of control functions (tasks) and means to ensure the implementation of these functions. The criterion for this choice is the optimal ratio between savings, revenues and the cost of the AS, eliminating

(C) Охунов Д.М., Охунов М.Х., 2021

САПР и моделирование в современной электронике. С. 107 - 110. 
unnecessary costs for the creation of the AS and significant management losses [2]. The essence of the choice task is to find solutions from a variety of possible options that best correspond to a particular AS.

The following approach is proposed to ensure the systematic evaluation and selection of AS components.

1. To build an organizational and functional model of the complex and its constituent individual local AS, in which, with an indication of all the relationships, all the functions performed by the relevant management body implementing the objectives of the object in all modes of operation with internal and external relationships are reflected.

2. The allocation and formalization of tasks and their complexes is carried out, the space of task attributes is built, their ordering is carried out. Task parameters allow further selection of models and methods for solving problems, software, a set of technical means, etc.

3. For the formation of mathematical models, the formalization of management functions is carried out, i.e. economic and mathematical models are built and the possibilities of their integration into blocks are determined. When constructing models for some AS, it is necessary to rely on the results of theoretical research in this area, as well as apply the experience of using models in other AS, primarily those where similar tasks are solved. When analyzing models, their characteristics such as: functional purpose, economic and mathematical properties, degree of development, etc. are taken into account.

One of the main goals of the analysis of economic and mathematical methods and models in each AS should be the creation of a bank of models designed to facilitate the task of finding and selecting appropriate economic and mathematical methods and models.

4. Selection of a set of algorithms for solving problems and programs that implement a given solution algorithm, AS technical means.

The general approach to the implementation of the tasks of selecting the components of the AS is formed as follows.

Let there be a set of alternative solutions $\left\{P_{i}\right\}$. It is necessary to choose a solution $\left\{\mathrm{P}_{\mathrm{k}}\right\}$, that satisfies the formulated requirements and limitations. Moreover, the result of such a choice may not be one solution, but a certain set of $\left\{\mathrm{P}_{\mathrm{j}}\right\} \subset \mathrm{P}$, since it is possible that several components of the same name with different characteristics are used in one speaker.

The decision-making process begins with the formulation of the problem, the definition of the objective function of choice, then the analysis is carried out and the ways to achieve the goal are formed that satisfy some given constraints and represent the initial set of alternative solutions formed in one way or another based on information about a particular object.

When forming a set of criteria for comparing alternatives, the consequences of each decision are revealed. The formation of a list of criteria is a complex iterative procedure, the success of which depends on how complete information about the object and its connections with the environment the developers of the AS have. 
The objective function of the system can be cost, time or resource type: minimum design costs; minimum design complexity, minimum time for the development and implementation of AS; minimum operating costs, including machine time; minimum loading of technical means; minimum system response to the question; minimum capital costs for the creation of AS, etc.

After defining the objective function, restrictions are set:

a) resource constraints. Under the resources (R) for the creation and design of the automated AS, information and computing services (ICS) are understood:

- cost costs of design;

- complexity of project work;

- the costs of operating the AS and the ICS system;

- capital expenditures, which include the costs of purchasing a complex of technical means (CTM), communications equipment, construction, modernization and repair of buildings and structures and laying communication channels, infrastructure creation, etc.

The resource required to create an AS and an ICS system and carry out design work should not exceed a certain pre-set value $\overline{R_{k}}$.

$$
R_{k} \leq \bar{R}_{k}
$$

b) time constraints associated with the creation of the AS and the ICS system and the timing of design work; the total duration of the process of creating and designing the AS, creating the ICS system, approved in accordance with the established procedure; the duration of individual stages of creating the AS and ICS and design work.

c) restrictions related to the required machine time.

d) logical constraints. They reflect the organizational, informational, technical and technological interrelationships of various components when choosing solutions, i.e. determine their compatibility.

The compatibility conditions of the decisions made can be set as follows:

1) the expressionmm $\chi_{m}+\chi_{n} \leq 1$ indicates the incompatibility of the decisions taken in the case when $\chi_{\mathrm{m}}$ and $\chi_{\mathrm{n}}$ correspond to the solutions of $\mathrm{R}_{\mathrm{m}}$ and $\mathrm{P}_{\mathrm{n}} \subset\left\{P_{i}\right\}$

2) the expression $\sum_{i=1}^{J}=1$ shows that only one solution out of the set $\left\{P_{i}\right\}$ can be used when creating an AS and an ICS and designing an AC.

The next step is to evaluate alternatives based on the selected criteria and the rating scale. As a result of constructing evaluation scales and evaluating many alternatives on these scales, a lot of estimates are obtained and the problem of choice is solved.

Evaluation and selection of solutions can be carried out by one of the following methods: direct calculations, statistical estimates, expert assessments, experimental verification and modeling. 
Practical evaluation of decisions made at specific facilities is carried out using all evaluation methods. At the same time, the practical combination of the methods discussed above is determined by the specific nature of the decisions taken.

For effective implementation and selection of options, it is advisable to develop a special (expert) system based on human-machine methods for solving selection problems, when the solution is achieved with human participation at certain stages of the iterative process implemented by a computer. The decision is made by a group of expert specialists in the "man-machine" dialogue mode.

The system should ensure the process of setting selection tasks, including the formation of a set of criteria and constraints, a set of estimates, the preparation of the necessary information, the choice of a model and a solution method based on the type of selection tasks, the solution of the selection task, as well as the means of collecting and processing information to ensure the collection of the necessary information, the analysis of the developed option and the choice of solutions.

The creation of thematic data banks characterizing automation objects, control functions and means of their implementation, and saturating them with sufficiently complete, reliable and operational information can be considered as a separate information valuable product.

The most complex element of the decision selection system, which is its core, is the BAP - bank of algorithms and decision selection procedures. The formation of the BAP is the process of creating a knowledge bank and requires significant experience from the developer in such areas of knowledge as theory and practice of management activities, information technology, as well as the development of expert systems.

\section{References}

1. S.S. Gulyamov, B.Y. Khodiev, A.A. Musaliev, B.A. Begalov. Information systems management: - Tashkent, TSEU. $2007-333$ p .

2. Okhunov D.M. Theoretical and methodological aspects of the development of marketing automated information systems. - Tashkent, Fan, 2020-208 p. 